Copyright (2012) Acoustical Society of America. This article may be downloaded for personal use only. Any other use requires prior permission of the author and the Acoustical Society of America. The following article appeared in:

Giordano, Bruno L.; Visell, Yon; Yao, Hsin-Yun; Hayward, Vincent; Cooperstock, Jeremy R.; and McAdams, Stephen. Identification of walkedupon materials in auditory, kinesthetic, haptic, and audio-haptic conditions. The Journal of the Acoustical Society of America, vol. 131, no. 5, pp. 40024012 (2012). DOI: $\underline{\text { https://doi.org/10.1121/1.3699205 }}$

and may be found at:

https://asa.scitation.org/doi/10.1121/1.3699205 


\title{
Identification of walked-upon materials in auditory, kinesthetic, haptic and audio-haptic conditions ${ }^{\text {a) }}$
}

\author{
Bruno L. Giordano ${ }^{\text {b) }}$ \\ Centre for Interdisciplinary Research in Music Media and Technology (CIRMMT), Schulich School of Music, \\ McGill University, 555 Sherbrooke Street West, Montréal, Quebéc, Canada H3A 1 E3. \\ Yon Visell \\ Centre for Interdisciplinary Research in Music Media and Technology (CIRMMT), Centre for Intelligent Machines \\ (CIM), McGill University, 3840 University Street, Montréal, Quebéc, Canada H3A 2 A\%. \\ Hsin-Yun Yao \\ Tactile Labs, Saint-Bruno, Québec, Canada \\ Vincent Hayward \\ Institut des Systèmes Intelligents et de Robotique, UPMC Université Paris 06, UMR 7222, 4 place Jussieu, \\ 75005 Paris, France \\ Jeremy R. Cooperstock \\ Centre for Interdisciplinary Research in Music Media and Technology (CIRMMT), Centre for Intelligent Machines \\ (CIM), McGill University, 3840 University Street, Montréal, Quebéc, Canada H3A 2A\%. \\ Stephen McAdams \\ Centre for Interdisciplinary Research in Music Media and Technology (CIRMMT), Schulich School of Music, \\ McGill University, 555 Sherbrooke Street West, Montréal, Quebéc, Canada H3A 1E3.

\begin{abstract}
Locomotion generates multisensory information about walked-upon objects. How perceptual systems use such information to get to know the environment remains unexplored. The ability to identify solid (e.g., marble) and aggregate (e.g., gravel) walked-upon materials was investigated in auditory, haptic or audio-haptic conditions, and in a kinesthetic condition where tactile information was perturbed with a vibromechanical noise. Overall, identification performance was better than chance in all experimental conditions and for both solids and the better identified aggregates. Despite large mechanical differences between the response of solids and aggregates to locomotion, for both material categories discrimination was at its worst in the auditory and kinesthetic conditions and at its best in the haptic and audio-haptic conditions. An analysis of the dominance of sensory information in the audio-haptic context supported a focus on the most accurate modality, haptics, but only for the identification of solid materials. When identifying aggregates, response biases appeared to produce a focus on the least accurate modality - kinesthesia. When walking on loose materials such as gravels, individuals do not perceive surfaces by focusing on the most accurate modality, but by focusing on the modality that would most promptly signal postural instabilities.
\end{abstract}

PACS numbers: 43.66.Lj,43.66.Wv,43.66.Jh,43.66.Ba

\section{INTRODUCTION}

Perceiving objects in the environment is one of the fundamental functions of sensory systems. Various studies reveal that humans are capable of perceiving object properties even in the absence of visual information when passively hearing sound-generating objects (Lutfi, 2007), when manipulating them in a purely haptic or active touch context, and in an audio-haptic context (Lederman and Klatzky, 2004). The study of the auditory and haptic perception of object properties has focused on manually generated events (e.g., hitting and scraping) and has left largely unexplored a highly ecological action that generates rich multisensory information about the envi-

\footnotetext{
a) Portions of this research were presented at Acoustics '08 Paris (Giordano et al., 2008).

b) Electronic address: bruno.giordano@music.mcgill.ca
}

ronment: locomotion. It is thus notable that although footstep sounds are among the non-speech sounds with the highest ecological frequency (Ballas, 1993, Appendix B), they have been investigated only by a handful of studies (e.g., Li et al., 1991; Pastore et al., 2008). This study aims to assess the extent to which non-visual information generated during locomotion contributes to our knowledge of objects in the environment. More specifically, we aimed to: (i) measure the ability to identify and discriminate walked-upon materials in various non-visual conditions (auditory, kinesthetic, haptic and audio-haptic); (ii) ascertain what sensory modality dominates identifications in audio-haptic conditions; (iii) ascertain the extent to which sensory dominance is determined by response biases (e.g., tendency to answer "A" more frequently than "B" in an experiment in which A and B stimuli are equally frequent). From the methodological standpoint, the measurement of kinesthetic perception in this study relied on a novel technique for disrupting tactile material-related information based on a vibrome- 
chanical masker. The masking approach is a viable alternative to previously adopted invasive methods based on hypothermia or pharmacology (Srinivasan and LaMotte, 1995; Perry et al., 2001).

Motor activity generates a great deal of multisensory information about objects in the environment. For instance, manipulation produces mechanical responses in objects that can stimulate the tactile and kinesthetic systems, which constitute the sense of active touch (haptics), and the auditory system. The hand thus serves a dual function: sensing and manipulation (Jones and Lederman, 2006). The stepping foot, like the manipulative hand, is a crucial link in the sensorimotor locomotion loop and is characterized by a dual function: sensing and support/traction. Stable and efficient locomotion is known to require the gathering of information about the ground serving as support (Ferris et al., 1998), and a large amount of sensory information is available for this purpose. Walking on a surface usually produces strong mechanical responses and, depending on the sole and ground materials, clearly audible acoustic emissions: the forces of interaction have a magnitude that is at least equal to the weight of a person. For example, when a shoe steps onto a solid material (e.g., marble), it produces transient interaction forces generated by impacts between the shoe sole and the floor surface. Previous studies of auditory and audio-haptic objects suggest that the spectrotemporal nature of these impacts contains information about the hardness of the ground material (DiFranco et al., 1997; Giordano et al., 2010b). The impulsive nature of the solid-ground signals can be contrasted with the temporally extended nature of those generated when stepping onto loose aggregate ground materials (e.g., sand): impacts may be less defined and can be accompanied by high-frequency textural components produced through processes such as the fracture of brittle structures and the inelastic displacement of load-bearing ground components (Galbraith and Barton, 1970; Ekimov and Sabatier, 2006, 2008; Visell, 2011). Research on the audio-haptic perception of the size of granules inside a container (Pittenger et al., 1997) suggests that walkers can perceive the size of the aggregate-ground elements based on the properties of the acoustical and vibromechanical signals.

A relatively small number of studies has assessed the perception of non-visual walking events and, in particular, of walked-upon objects. Focusing on audition, only two studies have assessed the perception of walker characteristics such as gender and posture ( $\mathrm{Li}$ et al., 1991; Pastore et al., 2008), whereas several studies have assessed the perception of various properties of mechanically excited objects such as their size, hardness, material class, and manner of excitation (e.g., Warren and Verbrugge, 1984; Freed, 1990; Grassi, 2005; Giordano and McAdams, 2006; Lutfi, 2007; Giordano et al., 2010b). This asymmetry is perhaps less evident within the haptics literature: although the perception of the properties of manually explored objects has been frequently investigated (e.g., weight, geometry, texture, compliance, Klatzky et al., 1985; Klatzky and Lederman, 2002; Ballesteros and Heller, 2008), several studies have assessed the perception of surface attributes that directly impact loco- motion and balance (e.g., slipperiness and slant, compliance, discriminability of tactile ground surface indicators, Kinsella-Shaw et al., 1992; Ferris et al., 1998; Donelan et al., 2002; Pan et al., 2005; Kobayashi et al., 2008; Rosburg, 2008; Courtney and Chow, 2000). However, none of these studies has focused on the perception of highly heterogeneous ground surfaces such as those encountered on a daily basis.

An overarching issue in the study of object perception is how information from different sensory modalities is combined in a multisensory context: which modality has the strongest influence? Does the availability of information from multiple modalities improve perceptual accuracy? Is multisensory perception dominated by the most accurate modality? To what extent do response biases modulate modality dominance? Our current knowledge of multisensory walking events is lacking in these respects because previous studies focused on those attributes of the environment that subserve navigation and disregarded information about object properties (e.g., Perry et al. 2001; Souman et al., 2009; see Visell et al., 2011, for a notable exception to this trend). In contrast, several previous studies investigated the perception of objects in non-walking audio-haptic interactive conditions. Overall, these studies revealed that although auditory information can modulate the haptic perception of object properties (e.g., crispness of potato chips, roughness of tactile textures, stiffness of tapped objects, wetness and roughness of rubbed hands, Zampini and Spence, 2004; Spence and Shanker, 2010; Kim et al., 2007; Suzuki et al., 2008; DiFranco et al., 1997; Avanzini and Crosato, 2006; Reyes-Lecuona and Cañadas-Quesada, 2009; Jousmäki and Hari, 1998; Guest et al., 2002), haptic information allows more accurate perception than audition (Lederman, 1979; Jansson, 1993; Pittenger et al., 1997; Pittenger and Mincy, 1999; Heller, 1982), and dominates auditory information in audio-haptic contexts (Lederman, 1979; Lederman et al., 2002; Altinsoy, 2008; Giordano et al., 2010a).

Overall, the literature on audio-haptic object perception, and in particular on manually explored audio-haptic objects is not sufficient to outline a set of clear expectations concerning how audition, haptics and, within the haptic modality, touch and kinesthesia are combined to yield a multisensory percept of walked-upon materials. Firstly, physiological differences between the somatosensory systems in the hand and foot (e.g., Weinstein, 1968; Kekoni et al., 1989; Kennedy and Inglis, 2002; Well et al., 2003), and the direct vs. mediated contact between the skin and the object with manual exploration and walking with shoes, respectively, can result in differences in the processing of haptic information. Secondly, the mechanical responses of surfaces to walking are much stronger than those resulting from manual touch (it is difficult to walk stealthily on most surfaces, whereas silent manipulation of objects is relatively effortless) and are likely to produce sounds that are more intense than those generated during manual exploration (e.g., Lederman, 1979). Importantly, the reliance on auditory information in audio-haptic conditions is modulated by the intensity of touch-generated sounds (Lederman et al., 2002; Lederman and Klatzky, 2004). Finally, to our knowledge none 
of the previous studies on the audio-haptic perception of objects has disentangled the contribution of tactile and kinesthetic information.

This study assessed the identification of walked-upon materials (solids and aggregates) in various non-visual sensory contexts: auditory (passive listening to walking sounds), kinesthetic (walking with masking of sound information and vibromechanical perturbation of touch information); haptic (walking with masking of sound), and audio-haptic (walking). Analyses assessed: (i) identification performance; (ii) identification-based material discriminability (referred to as discriminability in the following) independent of response biases; (iii) the similarity of identification confusions and patterns of material discriminability from different conditions. This last group of analyses ascertained patterns of sensory dominance in the audio-haptic context and determined whether dominance is accounted for by modality-specific discrimination abilities or, instead, is produced by response biases. Analysis (ii) and part of analysis (iii) relied on measures of bias-independent material discriminability as derived from General Recognition Theory models of identification confusions (Ashby and Townsend, 1986).

\section{EXPERIMENT}

\section{A. Methods}

\section{Participants}

Five males participated in the experiment [average age $=25$ years, $\mathrm{SD}=4$ years; average weight $=74.9 \mathrm{~kg}$, SD $=4.7$; average height $=173.8 \mathrm{~cm}, \mathrm{SD}=1.8$; average shoe size (US) $=10, \mathrm{SD}=1.4]$. None of them reported motor or haptic deficits; all had normal hearing (ISO, 2004; Martin and Champlin, 2000).

\section{Apparatus}

The kinesthetic, haptic and audio-haptic conditions took place inside a mildly reverberant room with a wooden floor. The apparatus for these conditions is illustrated in Fig. 1. Curtains separated a response area from a ground-presentation area. Ground materials were installed on top of two $240 \times 120 \mathrm{~cm}$ plywood panels, each supported with sixteen heavy-duty rollers. The panels were displaced on top of a vibration-dampening layer of soft rubber. Wood strips divided each panel into four $60 \times 120 \mathrm{~cm}$ areas, each containing one ground sample (ground-to-floor height $\approx 10 \mathrm{~cm}$ ). Two $12 \mathrm{~cm}$-high platforms were placed on the opposite sides of the rubber sheet, one in the response area and one in the presentation area. They were covered with vinyl tiles and were laid on top of a vibration-dampening layer of soft rubber. Ropes hanging from wood poles were secured to the sides of the platforms, and delimited the walking perimeter in the presentation area. On each trial, a ground material was placed between the two platforms. Blindfolded participants walked on the ground sample while holding the

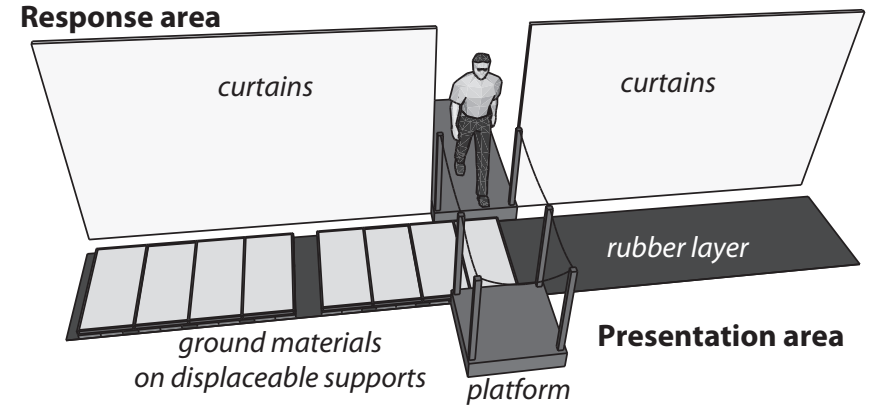

FIG. 1. Apparatus used for the presentation of the ground materials.

ropes with their hands. Participants wore appropriately sized rubber-sole leather shoes of the same model. A computer was used to collect the identification responses.

\section{[Insert Figure 1 about here]}

In the kinesthetic condition, walking participants were presented with a vibrotactile, pseudo-random noise signal meant to perturb tactile information. This was produced by an array of four tactile actuators for each shoe (two for the heel, two for the toe), each placed in the middle of a carbon-fiber tube installed in the top cork layers of a 25-mm outer sole. These recoil-type actuators were similar in design to those described by Yao and Hayward (2010). The orientation of the magnets inside the coil maximized the magnitude of displacements in the lateral direction with respect to the plantar surface of the foot. The outer sole was firmly strapped to the shoes worn by participants. The vibrotactile actuators were driven by a light-weight, $20 \mathrm{~W}$, battery-powered amplifier, connected to a portable media player, all placed inside a backpack. Participants used an external switch to turn the amplifier on and off as needed. In the audio-haptic condition, participants used the same outer sole as in the kinesthetic condition, but without vibrotactile actuators. This equalized eventual effects of the outer sole on the gait dynamics across conditions. Walking sounds generated during this condition were recorded with a Beringer ECM8000 microphone, feeding into a Motu 828mkII digital audio interface. The microphone was located $8 \mathrm{~cm}$ above the center of the edge of a given ground sample, pointing down at an angle of $22^{\circ}$. In the haptic condition, participants used the same unactuated outer sole as in the audio-haptic condition. Walking participants were presented with a continuous $94 \mathrm{~dB}$ SPL white noise (sampling frequency $=48 \mathrm{kHz}, 16$-bit resolution) over Sennheiser wireless Rs146 headphones. Participants wore ear plugs to prevent hearing damage (noise reduction rating $=29 \mathrm{~dB}$; ANSI, 1974).

For the auditory condition, sound stimuli were stored on the hard disk of a Macintosh G5 computer equipped with an M-Audio Audiophile 192 s/PDIF interface for digital-to-analog conversion. Audio signals were amplified with a Grace Design m904 monitor system and presented binaurally through Sennheiser HD280 headphones. Participants sat inside an IAC double-walled soundproof booth. Signal peak level at the headphones ranged from 
35 to $80 \mathrm{~dB}$ SPL, as measured with a Brüel \& Kjær Type 2205 sound-level meter coupled with a Brüel \& Kjær Type 4153 artificial ear. The level of reproduction corresponded approximately to that of the live sounds, as measured with a Brüel \& Kjær Type 2205 sound-level meter located approximately at the height of the head of the walker.

\section{Stimuli}

Walking-ground stimuli included four aggregate materials [very small gravel $(\varnothing 6 \mathrm{~mm})$, small gravel $(\varnothing 8$ $10 \mathrm{~mm}$ ), medium gravel $(\varnothing 13 \mathrm{~mm})$, and large gravel ( $\varnothing 16-20 \mathrm{~mm})$ ], and four solid materials [a different vinyl than the one used to cover the response- and presentation-area platforms, plywood, ceramic, and marble (from softest to hardest)]. The ground materials were selected based on the results of a preliminary identification experiment carried out with the same participants so as to increase the likelihood of identification confusions within the aggregate and solid categories while still focusing on materials encountered during everyday walking. Comparatively high confusion rates were required by the General Recognition Theory analysis of identifications.

In the auditory condition, participants were presented with recordings of their own walking sounds from the audio-haptic trials, including one preliminary audiohaptic session. For each of the materials, at least three recordings were selected after low-level signals and footdragging sounds were discarded. Each sound stimulus included two initial steps on the walking ground, followed by the steps taken on the platforms while the participant turned back toward the ground material, and two final steps on the walking ground. The tactile masker presented during the kinesthetic condition was a pseudorandom noise (sampling frequency $2.0 \mathrm{kHz}$ ) synthesized to maximize the spectral level of the lateral acceleration (see Fig. 2, for details).

\section{[Insert Figure 2 about here]}

The vibromechanical masking stimulation to the feet amounts to very small oscillatory movements, much smaller than $1 \mathrm{~mm}$, that could not have had any direct mechanical consequences on posture and gate. In the kinesthetic condition, exposure to the tactile masker was limited to when participant walked on the ground stimuli and was not sufficient to numb the feet. The masking signal was highly attenuated at frequencies below $100 \mathrm{~Hz}$, and thus primarily masked stimuli transduced by subclasses of cutaneous tactile receptors exhibiting a higher-frequency, phasic response (fast-adapting receptors), which are most sensitive to transient or vibrating stimuli. The masker would have had little effect on the slow-adapting receptors that preferentially respond to slowly varying stimulation, notably the quasi-static foot-ground forces that are most important for regulation of balance and locomotion. Thus, the procedure minimized the likelihood of adaptation effects of tactile receptors, and subsequent effects on posture and gait. In sum, the consequences of the masking stimuli are pre-
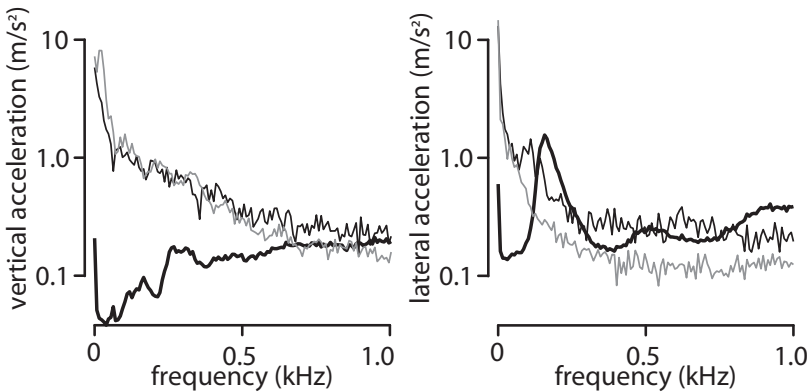

FIG. 2. Vertical and lateral acceleration spectra at the center of the toe for the vibrotactile noise (thick black line), and for the wood and small gravel grounds (thin grey and black lines, respectively; sampling frequency $=2 \mathrm{kHz}$; FFT size $=256$ samples). Measurement equipment: $\mathrm{PCB} 352 \mathrm{C} 42$ accelerometer connected to a Model 480E09 PCB signal conditioner itself connected to a National Instruments USB-6218 board for analog-to-digital conversion.

dominantly sensory.

\section{Procedure}

On each trial, participants were presented with one of the eight grounds and were asked to identify the material by clicking with the mouse on one of eight on-screen buttons labeled "vinyl", "wood", "ceramic", "marble", "very small gravel", "small gravel", "medium gravel" or "large gravel". Response time was not limited, and feedback on identification performance was never given. Each participant identified the walking grounds in each of the kinesthetic, auditory, haptic, and audio-haptic conditions.

At the beginning of each haptic and audio-haptic trial, participants stood blindfolded on the response-area platform. At the signal of the experimenter, they opened the curtains, took two steps on the walking ground, turned around while on the presentation-area platform, took two more steps on the walking ground, reached the responsearea platform, closed the curtains and removed the blindfolds. Participants were instructed to walk with a normal pace and to avoid dragging their feet on the ground. The walking ground for the next trial was put in position while participants were in the response area. In order to mask the sounds generated while preparing the next trial, as long as participants remained in the response area they were presented with a 94 or $70 \mathrm{~dB}$ SPL white noise in the haptic and audio-haptic conditions, respectively (participants in the audio-haptic condition did not wear ear plugs). The procedure for the kinesthetic condition was the same as for the haptic condition, with the difference that participants started the playback of the vibrotactile noise immediately before taking the first step on the ground material and terminated it immediately after returning to the response-area platform. On each trial of the auditory condition, participants heard one recording of the target material chosen at random from among the available samples. They could listen to 
the sound stimulus only once. In the kinesthetic, haptic and audio-haptic conditions, they were shown labeled samples of the walking grounds arranged on a table located in an unreachable position of the response area. Participants were not allowed to touch them. Participants in the auditory condition were shown a picture of the same labeled ground samples.

Each individual took part in twelve experimental sessions on different days, divided into three blocks of four sessions each (one session per condition). The order of presentation of the experimental conditions was randomized within each block of four sessions. On each experimental session, the eight walking grounds were presented in random order on each of eight blocks of trials, for a total of 64 trials. Each session lasted approximately 90 minutes. All participants identified the same walking grounds as in the current experiment during a short preliminary audio-haptic session, meant to collect recordings for the auditory conditions, and in one additional preliminary experiment (conditions: haptic, auditory, and audio-haptic) meant to select the stimuli for the current experiment, and to allow participants to adapt to the experimental procedure and apparatus (e.g., walking blind with shoes slightly higher than usual). The set of stimuli in this preliminary experiment included half of the walking grounds investigated in the current experiment. No feedback on performance was given.

\section{B. Results}

Table I reports the matrices of identification confusion in the population of participants in each of the experimental conditions. Solid materials were rarely confused with aggregates and vice versa (grand average proportion of confusion errors across experimental conditions $=.015$, range across participants $=.00-.03)$. The patterns of identification confusion also reveal an influence of the hardness of solid materials and of the size of the gravel. For each participant and for each experimental condition, the confusion probabilities were rank-correlated with the absolute difference in the ranked hardness or gravel size between each pair of either solid or aggregate materials (e.g., difference between very small gravel and large gravel $=4)$. For each condition and material category (solid vs. aggregates), a paired t-test was used to assess whether the average correlation $\rho$ in the population of participants was significantly different than zero. The t-tests were carried out on the Fisher-Z transformed correlations (Fisher, 1915). For both material categories, and in all experimental conditions, confusions were less likely between materials of highly diverse hardness or gravel size (grand-average $\rho$ $=-.540, \mathrm{SD}=.434)$. In particular, the association between identification confusions and material properties was significant for aggregates in all experimental conditions $[\rho \leq-.647, t(4) \leq-4.16, p \leq .014]$, whereas for solids a significant association emerged only in the haptic condition $[\rho=-.555, t(4)=-3.37, p=.028$; $\rho \geq-.301, t(4) \geq-2.59, p \geq .061$ for the other experimental conditions]. Subsequent analyses considered

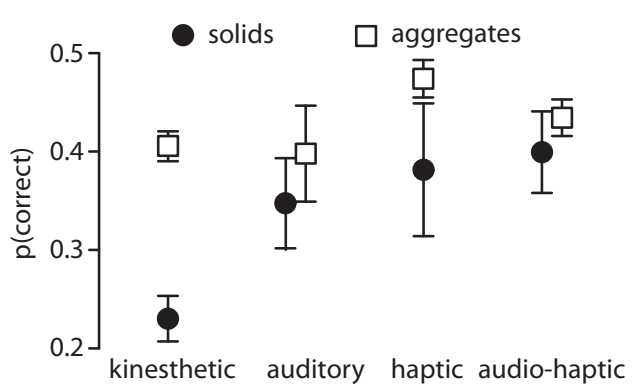

FIG. 3. Average identification performance for each material category and experimental condition. Error bar: \pm 1 SEM.

the matrices of identification confusions within the categories of solid and aggregate materials and assessed: [1] identification performance; [2] bias-independent discrimination abilities; [3] the similarity of the patterns of identification confusions and discriminability among different experimental conditions.

\section{[Insert Table I about here]}

\section{Identification performance}

Analyses tested for better-than-chance identification performance and assessed the effects of experimental condition and material category (solids vs. aggregates) on performance (Fig. 3: average condition- and categoryspecific performance). Considering a chance performance of $12.5 \%$ correct, performance was better than chance in all experimental conditions and for both material categories [single-sample $t(4) \geq 4.24, p \leq .007$ ]. Because of the almost perfect ability to distinguish between solid and aggregate materials, a more appropriate measure of chance-level performance would be to focus on the ability to identify materials within the solid and aggregate categories (chance performance $=25 \%$ correct). Performance levels higher than $25 \%$ correct were observed for all experimental conditions and material categories [single-sample $t(4) \geq 2.18, p=.048$ ], with the exception of the performance for solids in the kinesthetic condition $[t(4)=0.91, p=.792]$.

\section{[Insert Figure 3 about here]}

The effect of experimental condition and material category on identification performance was assessed within a linear mixed-effects model (LMM, Verbeke and Molenberghs, 2000; West et al., 2006) fitted using the SAS ${ }^{\circledR}$ PROC MIXED routine (Littell et al., 2006). In general terms, LMMs can potentially include both fixed and random effects measuring the average effect and its variability within a unit of interest (e.g., population), respectively. With LMMs, both the fixed and random effects and the structure of the model covariances are the object of model selection procedures (West et al., 2006, pp. 3941). The LMMs presented in this study have a simplified structure: they include both significant and nonsignificant fixed effects, have a random effect for the intercept, modeling the variation of the average of the participantspecific data across individuals, and adopt a compound- 
TABLE I. Matrices of identification confusions in the population of experimental participants (rows: stimuli; columns: responses). $\mathrm{MA}=$ marble; $\mathrm{CE}=$ ceramic; $\mathrm{WO}=$ wood; $\mathrm{VI}=$ vinyl $; \mathrm{VG}=$ very small gravel; $\mathrm{sG}=$ small gravel; $\mathrm{MG}=$ medium gravel; $\mathrm{LG}=$ large gravel. Number of repeated presentations of each material in each experimental condition $=120$.

Experimental condition

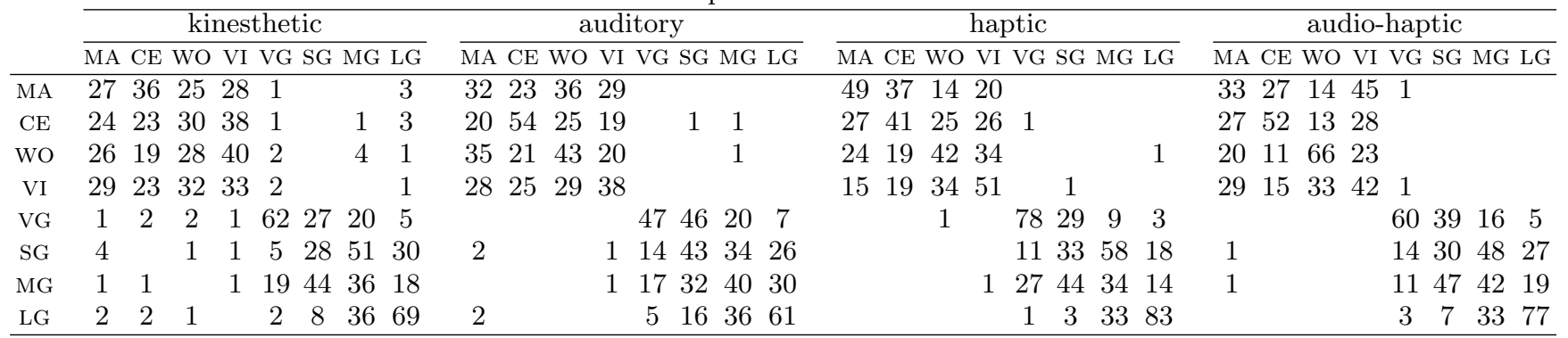

symmetry structure for the individual-specific covariance matrix of the residuals. Conclusions warranted by more complex models, resulting from model-selection procedures, were equivalent to those warranted by the simplified models presented here. All the models presented here satisfied the normality assumption for participantspecific residuals [Shapiro-Wilk $W \geq .95, p \geq .281$ ].

Identification performance was thus analyzed within an LMM that included the fixed effects of experimental condition, material category, condition $\times$ category, and ground material(category), i.e., material nested within category (the factor ground material is nested within the factor material category because, for instance, "material" is "marble" only when "category" is "solid"). The material(category) effect was significant $[F(6,142)=$ $14.02, p<.001]$, indicating at least one significant difference in the identification performance for two materials in the same category. The condition $\times$ category effect was not significant $[F(3,142)=2.21, p=.090]$. The effect of category was significant $[F(1,142)=17.04, p<.001]$, indicating better identification performance for aggregates than for solids. Finally, the effect of experimental condition was significant $[F(3,142)=5.52, p=.001]$, with significantly worse performance in the kinesthetic condition than in the haptic and audio-haptic conditions, $[t(142) \leq-3.32$, Bonferroni-adjusted (Bonf.-adj.), $p \leq .001 ; \operatorname{abs}(t(142)) \leq 1.83$, Bonf.-adj., $p \geq .069$ for the other post-hoc pairwise contrasts between experimental conditions].

\section{Discriminability}

Measures of the bias-independent ability to discriminate the ground materials were derived from General Recognition Theory (GRT, Ashby and Townsend, 1986; Ashby and Perrin, 1988; Ashby, 1992) models of identification data. GRT is a multidimensional extension of signal detection theory (SDT, Green and Swets, 1966) capable of analyzing confusion matrices from multi-category identification experiments characterized by strong response biases. It models the perceptual effects $f_{A}(\cdot)$ for the stimulus $S_{A}$ as a multivariate normal distribution with given mean and covariance matrix. In an identification experiment, participants are assumed to partition the space of sensory effects into independent regions using decision boundaries that are often modeled as a linear function of the space dimensions. Perceptual effects that fall within the same region will receive the same identification response $R$. Assuming a two-dimensional sensory space with dimensions $x$ and $y$, the probability $p\left(R_{B} \mid S_{A}\right)$ that $S_{A}$ will receive the response $R_{B}$ corresponds to:

$$
p\left(R_{B} \mid S_{A}\right)=\int_{r_{B}} \int f_{A}(x, y) d x d y
$$

where $r_{B}$ is the region of the space associated with response $R_{B}$. GRT emphasizes the notion of betweenstimuli proximity rather than that of between-stimuli distance. The bias-independent proximity between two stimuli is termed similarity, $s$, and is defined as

$$
s\left(S_{A}, S_{B}\right)=\int_{f_{A}<f_{B}} \int f_{A}(x, y) d x d y, s \in[0,1],
$$

where $f_{A}<f_{B}$ denotes the region of the space where the sensory effects for $S_{A}$ are less likely than those for $S_{B}$. The GRT measures $s$ of sensory similarity are thus analogous to $d^{\prime}$ in SDT. Within the current study, these measures can be interpreted as a response-bias-free measure of the ability to discriminate ground materials based on identification responses, where $s=0$ and 1 for perfectly accurate and perfectly inaccurate discrimination, respectively.

\section{[Insert Figure 4 about here]}

One GRT model was estimated for each of the participants in each of the experimental conditions. The almost perfect solid-aggregate discrimination resulted in around half of the 64 cells in each confusion matrix having a value of zero. For this reason, a maximum of 32 degrees of freedom were available for model fitting. The number of parameters in each GRT model was thus reduced to 26 by assuming: (i) a two-dimensional space (two location parameters for the mean of each stimulus distribution); (ii) the same covariance matrix for materials within the same category (three parameters for each of the two covariance matrices); (iii) two fixed orthogonal decision boundaries with one of them separating the two material categories (no free parameter); (iv) two free decision boundaries, one for the solids and one for the aggregates (two parameters for each boundary). Model fitting was based on 


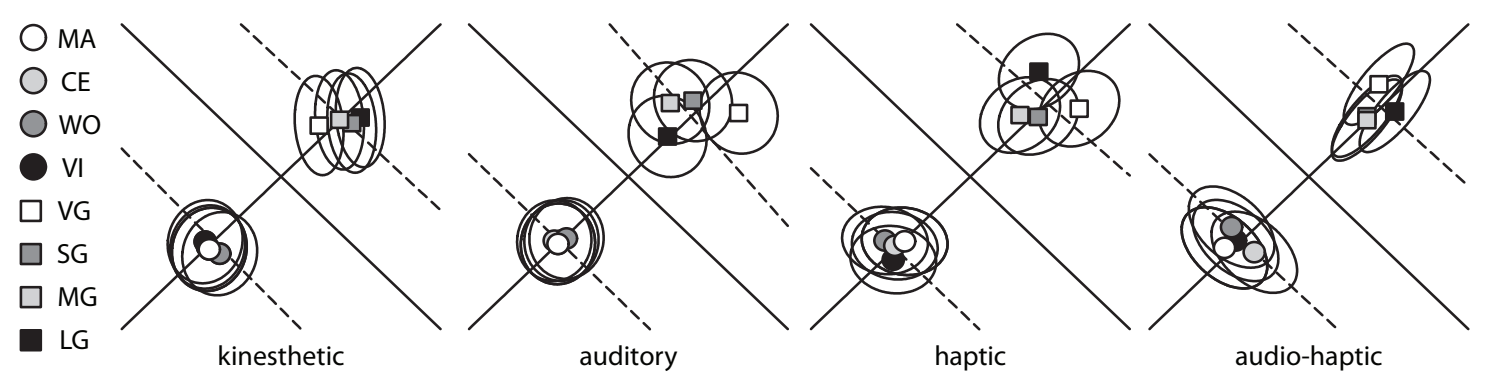

FIG. 4. GRT models of the identification confusions for one of the participants in each of the experimental conditions. A bivariate normal distribution models the across-trials fluctuations in the sensory effects of a ground material (symbols $=$ means; ovals $=0.05$ equal-likelihood contours). Lines model the decision boundaries set by participants to carry out the identification task ( solid lines = fixed boundaries; dashed lines = boundaries fitted to experimental data). Sensory effects that fall within the same region of the two-dimensional decision space receive the same identification response. Materials are labeled as in Table I.

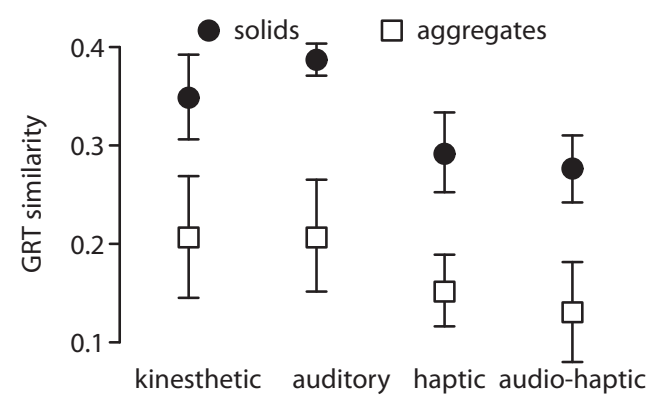

FIG. 5. Average condition-specific GRT similarity between materials in the same category. Similarity $=0$ and 1 for perfectly accurate and inaccurate discrimination, respectively. Error bar: \pm 1 SEM.

the method described in Ennis and Ashby (2003), combined with an iterative routine for the minimization of the sum of the squared model-prediction errors (SSE). The starting configuration used by the SSE minimization routine was the same for all confusion matrices: the free decision boundaries were parallel to the solid-aggregate boundary; the mean of the stimulus distributions was located at the intersection of the free boundary with the fixed boundary being orthogonal to the solid-aggregate boundary; all the covariance matrices were an identity matrix. Thus constrained and fitted, the GRT models accounted well for the data in all the confusion matrices $(\mathrm{SSE}=0.004-0.090 ; \mathrm{RSQ}=.910-.996)$. Fig. 4 shows the GRT models for one representative participant in each of the experimental conditions. Fig. 5 shows the average between-stimuli GRT similarity for solid and aggregate materials in each of the conditions.

\section{[Insert Figure 5 about here]}

GRT similarities were analyzed within an LMM with the fixed effects of experimental condition, material category, condition $\times$ category and materials-pair(category). Only GRT similarities for materials within the same category were considered. The materials-pair(category) effect was significant $[F(10,218)=8.20, p<.001]$, indicating that at least two of the similarities within the same category were different. The condition $\times$ category effect was not significant $[F(3,218)=0.65, p=.585]$. The effect of category was significant $[F(1,218)=179.67, p<.001]$, indicating a better ability to discriminate among aggregates than among solids. Finally, the effect of experimental condition was significant $[F(3,218)=15.55, p<$ $.001]$. In particular, all post-hoc pairwise contrasts between average condition-specific similarities were significant $[\operatorname{abs}(t(218)) \geq 3.45$, Bonf.-adj., $p \leq .001]$, with the exception of no significant difference between the auditory and kinesthetic conditions, and between the haptic and audio-haptic conditions $[\operatorname{abs}(t(218)) \leq 1.24$, Bonf.adj., $p \leq .228]$.

\section{Comparison of experimental conditions}

A last analysis compared the patterns of identification confusions or the bias-independent GRT measures of discriminability from the different experimental conditions in order to: (i) assess significant between-condition similarities; (ii) analyze the variation of the betweencondition similarity across material categories and data types (identifications vs. discriminabilities); (iii) assess significant patterns of sensory dominance in the audiohaptic condition; (iv) establish whether the sensory dominance was accounted for by discrimination measures, as based on the assessment of which among the non-audiohaptic conditions yielded identifications or discriminabilities that most strongly resembled audio-haptic data.

The similarity between condition-specific data was quantified in terms of the concordance correlation $\rho_{c}$ (Lin, 1989), a special case of the Pearson correlation that measures the departure from the equality line $\left(\rho_{c}(A, B)=1\right.$ and -1 if $A=B$ and $A=-B$, respectively). Focusing either on the identification confusions or on the discriminabilities, one $\rho_{c}$ coefficient was computed to compare the data for the same participant from each pair of experimental conditions (6 pairs) and for each of the solid and aggregate categories, for a total of $24 \rho_{c}$ coefficients for each participant. The comparison of confusion matrices did not consider the correct responses in order to minimize the effects of identification performance and aid an interpretation of results in terms of response biases. The comparison of discriminabilities did not consider the self-similarities (e.g., 


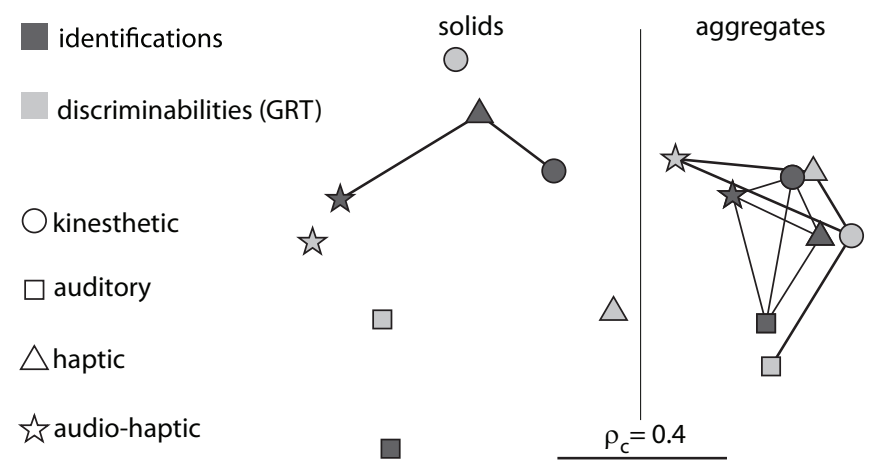

FIG. 6. Metric MDS models of the $\rho_{c}$ measures of betweencondition similarity for each data type and material category (lines connect significantly similar conditions, $p<.05$ ). Conditions that lie closer within the MDS configuration yield highly similar data (see scale in figure). A Procrustes rotation was used to align the MDS models for identifications and discriminations for the same category (scale factor for rotation $=0$ ). The MDS distances between different data types and categories are meaningless.

similarity between marble and itself) which, by definition, equal 1. All $\rho_{c}$ measures were Fisher-Z transformed (Fisher, 1915) prior to conducting any statistical analysis and modeling. Fig. 6 displays the two-dimensional metric multidimensional scaling (MDS) model of each of four $\rho_{c}$ matrices of condition similarity averaged across participants: identifications-solids; discriminabilities-solids; identifications-aggregates; discriminabilities-aggregates. The MDS models were fit to a distance between experimental conditions defined as $1-\rho_{c}\left[R^{2} \geq 0.95\right.$ across the four $\rho_{c}$ matrices].

[Insert Figure 6 about here]

Significant between-condition similarities were assessed based on a one-tailed one-sample t-test that ascertained whether the across-participant average of each $\rho_{c}$ coefficient was significantly higher than zero. For solid materials, no significant similarity emerged between the discriminabilities for the different experimental conditions $[t(4) \leq 1.67, p \geq .086]$, and significant similarities between identification confusions emerged only between the haptic and kinesthetic and between the haptic and audio-haptic conditions $[t(4) \geq 3.43, p \leq .013$; $t(4) \leq 1.53, p \geq .010$ for the other comparisons]. For aggregate materials, and for both the discriminabilities and identifications, all between-condition similarities were significant $[t(4) \geq 2.23, p \leq .044]$, with the exception of the similarity between the discriminabilities for the auditory condition, on the one hand, and the haptic and audio-haptic conditions, on the other $[t(4) \leq 1.94$, $p \geq .062]$.

The $\rho_{c}$ measures of between-condition similarity were analyzed within an LMM that included the fixed effects of material category, data type (identifications vs. discriminabilities), pair of experimental conditions (e.g., auditory-haptic), and all the possible interactions between these factors. The three-way interaction was significant, indicating that whereas for solids identifications in the kinesthetic and haptic conditions were

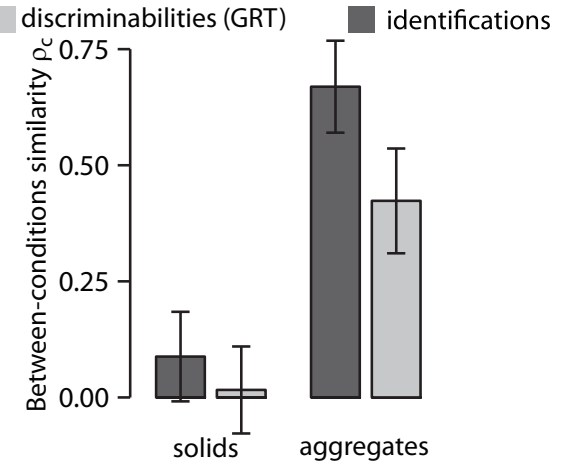

FIG. 7. Similarity (average $\rho_{c}$ ) of identification confusions and GRT discriminabilities for solid and aggregate materials. Error bar: \pm 1 SEM.

more similar to each other than the discriminabilities for these conditions $[F(1,92)=7.34$, Bonf.-adj. $p=.049$; $F(1,92) \geq 5.33$, Bonf.-adj. $p \leq .139$ for the other posthoc contrasts], aggregate identifications in the kinesthetic and multisensory conditions were more similar to each other than the discriminabilities for these conditions $[F(1,92)=14.71$, Bonf.-adj. $p=.001 ; F(1,92) \geq 4.92$, Bonf.-adj. $p \leq .174$ for the other post-hoc contrasts]. Both the main effect of pair and the data-type $\times$ pair interaction were significant $[F(5,92) \geq 2.74, \quad p \leq .024]$, indicating that not all the similarities between experimental conditions were equal, and that they varied depending on whether the experimental conditions were compared based on identifications or on discriminations. The main effects of material category and data type, as well as the interaction between these factors were significant $[F(1,92) \geq 6.27, p \leq .014]$ (see Fig. 7). For both identifications and discriminations, data from different experimental conditions were more similar for aggregates than for solids $[F(1,92) \geq 29.20$, Bonf.-adj. $p<.001]$. Whereas aggregates identifications from different conditions were more similar to each other than were discriminations $[F(1,92)=19.68$, Bonf.-adj. $p<.001]$, for solid materials the similarity of the identifications from different conditions was not significantly different than what observed for the discriminations $[F(1,92)=0.80$, Bonf.adj. $p=1.000]$.

\section{[Insert Figure 7 about here]}

A final analysis determined which among the kinesthetic, auditory and haptic conditions yielded data that most strongly resembled those for the audio-haptic condition. For each material category and for both identifications and discriminations, paired-sample t tests were used to ascertain significant pairwise differences among the $\rho_{c}$ coefficients between the audio-haptic condition, on the one hand, and all the other conditions, on the other. For aggregate materials, identifications in the kinesthetic condition resembled identifications in the audio-haptic condition more closely than those in any other condition $[t(4) \geq 4.68$, Bonf.-adj. $p \leq .028$; Bonf.-adj. $t(4)=-2.33, p=.240$ for the auditory vs. haptic comparisons]. For solid materials, no condition yielded data that most strongly resembled those for the audio-haptic 
condition $[\operatorname{abs}(t(4)) \leq 2.56$, Bonf.-adj. $p \leq .188]$. Interestingly, for both aggregate and solid materials no condition comparison involving the GRT discriminabilities revealed an experimental condition that yielded data that more strongly resembled those for the audio-haptic context $[\operatorname{abs}(t(4)) \leq 2.01$, Bonf.-adj. $p \leq .343]$.

\section{Discussion}

In all experimental conditions solid materials were seldom confused with aggregate materials. This result suggests either a large between-category diversity in sensory information (e.g., stepping on solids produces not more than two vibromechanical impacts; stepping on aggregates produces a sequence of temporally distributed impacts), or the fact that the perceptual system is well equipped for differentiating between solids and aggregates because of the high relevance of this distinction for the control of locomotion. After the nearly perfect differentiation between solid and aggregate materials was accounted for, better-than-chance identifications emerged for all the experimental conditions except for the solid materials in the kinesthetic condition. Intact touch information, which was perturbed in the kinesthetic condition, was thus strictly necessary for identifying solids but not for identifying aggregate materials. Similar effects of experimental condition emerged for both solids and aggregates, with significantly worse performance in the kinesthetic condition. It is unclear whether identification-performance results are the product of the discriminability of sensory information or of response biases (e.g., both better discrimination abilities and weaker response biases could produce superior identification performance for aggregates).

Bias-independent measures of material discriminability were estimated within the GRT framework. Consistent with a discrimination-based interpretation of the superior aggregate-identification performance, aggregates were better discriminated than solids in all experimental conditions. This result suggests the presence of more sensory information concerning the identity of aggregate materials than is the case with solids (e.g., with aggregates, information can be extracted from a larger number of impacts produced by the interaction of the heel and toe with multiple objects, and also from their temporal patterning, whereas with solids, information must be extracted from a lower number of impacts whose temporal patterning is largely independent of the ground material). Experimental condition affected similarly the discriminability of materials in both categories. This result is somewhat surprising because for mechanically different phenomena such as walking on solids and aggregates (see Introduction), discrimination abilities are not necessarily maximized or minimized by the same modalities. We have no clear explanation for this result. Importantly, a significant increase in discriminability was observed from the auditory and kinesthetic conditions to the haptic and audio-haptic conditions. Superior discrimination in the haptic and audio-haptic conditions would be expected because the auditory and kinesthetic conditions were both unimodal and thus likely poorer in sensory information. Interestingly, the same level of discriminability was observed for the haptic and audio-haptic contexts, i.e., adding auditory information to an haptic context produced weak gains in perceptual processing.

Overall, the analysis of discrimination is consistent with previous observations of a higher accuracy of haptic perception than auditory perception during the manual exploration of objects (Lederman, 1979; Jansson, 1993; Pittenger and Mincy, 1999). Contrary to what was observed for the measures of discriminability, the analysis of identification performance failed to support this hypothesis of higher accuracy. Response biases are likely the reason for this, because they influence identification performance but not discriminability. Finally, the fact that the perturbation of tactile information impaired discrimination down to the level observed for the auditory condition suggests that the superiority of haptic compared to auditory perceptual processing is, at least in part, due to the multisensory nature of the haptic modality.

A subsequent analysis assessed the similarity of the patterns of (bias-independent) discriminability and (biasdependent) identification confusions from different experimental conditions. The comparison of results for discriminabilities and identifications made possible to infer the effects of the response biases on the similarity of perceptual processes in the different conditions (e.g., response biases are likely the reasons for why conditions A and $\mathrm{B}$ yield different discriminabilities but similar identifications). The discriminations in different experimental conditions resembled each other more strongly for aggregate than for solid materials. This result is consistent with a greater cross-modal redundancy of sensory information for aggregate materials (information from different modalities is perfectly redundant if it affords the exact same pattern of discriminabilities). This result is evocative of the superior discriminability and identifiability of aggregate materials because redundant information across integral sensory dimensions (stimulus dimensions that can't be processed independently of each other) leads to superior perceptual performance (Ashby and Townsend, 1986; Klatzky et al., 1989). It is however important to recall that explanations for the superior processing of aggregate materials based on the crossmodal redundancy of information are of limited value because they don't explain why aggregates are also better identified than solids in the unimodal conditions. Further, this account would require a demonstration of crossmodal integrality of ground-identity information based on different experimental methods than those adopted here. Finally, identifications from different conditions were more similar to each other than were the discriminations only for aggregates but not for solids. This result suggests a strong cross-modal consistency of response biases for the identification of aggregate materials, which results in a greater similarity of identification data from different experimental conditions than is to be expected on the basis of discrimination alone.

The dominance of modality-specific information in the audio-haptic context was also assessed by comparing data from different conditions. For solid materials, only haptic 
identifications were significantly similar to audio-haptic identifications. Given the superior discriminability of materials in the haptic condition, this result thus appears consistent with the modality-appropriateness hypothesis according to which the dominant information in a multisensory context is the one that maximizes perceptual performance when presented alone (Welch and Warren, 1980). Note, however, that the support of the modality-appropriateness hypothesis is only partial. Indeed, more detailed pairwise comparisons among the similarities of the audio-haptic identifications to those in all the other conditions revealed no condition that yielded identifications significantly more similar to those in the audio-haptic condition. The same null result emerged when focusing on the patterns of discriminability.

Different results emerged for the aggregates. Most likely because of response biases that were strongly consistent across all conditions, audio-haptic identifications were significantly similar to identifications in all of the other conditions. Importantly, subsequent pairwise comparisons revealed that audio-haptic identifications more strongly resembled identifications in the kinesthetic condition than those in any other condition. Notably, the audio-haptic dominance of kinesthetic information was not explained by the similarity of discrimination patterns: the audio-haptic discriminabilities were equally similar to the discriminabilities in all the other conditions. Overall, the results for aggregate materials are in disagreement with what is predicted by the modalityappropriateness hypothesis: firstly, because the dominant information in the audio-haptic context affords the least accurate identifications and discriminations; secondly, because the dominance of the kinesthetic information appears to be a product of biases alone, i.e., is not explained by the patterns of material discriminability related to the quality of the sensory information. These results thus support the hypothesis that modality dominance is also influenced by modality-weighting biases developed in everyday multisensory contexts (Lederman and Klatzky, 2004). It is indeed plausible that everyday walking on aggregate materials promotes a focus on kinesthetic information because this modality is highly critical to the control of locomotion: when walking on materials such as gravel it is perhaps safest to focus on the sensory channels that best signal unstable locomotion (e.g., vestibular system, joint capsule receptors), rather than on those modalities that afford a finer discrimination of the gravel grain.

A few final considerations are necessary about the interpretation of the results for the auditory and kinesthetic conditions. The auditory condition was the only passive one (participants were presented with prerecorded stimuli). It might be speculated that walking participants allocate attentional resources more efficiently at foot impact time, because they actively control the locomotion program, and might thus be capable of better processing interactively produced sounds than recorded sounds. Further, the sound recordings were taken at around ground level, not at ear level, and did not contain binaural information or information about the filtering effects of the body and head. For these reasons, the estimates of auditory discrimination and identification performance might be at best an underestimate of what would be observed in a hypothetical active auditory condition in which normal walking with selective (and silent) suppression of kinesthetic and tactile information is possible. Note, however, that these interpretational caveats do not apply to the nonsignificant difference in perceptual performance between the haptic and audiohaptic conditions. Focusing on the kinesthetic condition, the exact origin of the observed effects of the vibromechanical masker is unknown. A working hypothesis was adopted according to which the vibromechanical noise selectively impaired the ability to process tactile information about ground identity. Further experimentation is necessary to establish the exact extent of the masking of tactile information, to assess the eventual effect of non-sensory factors (e.g., attentional resources), and to ascertain the potential effects of the vibromechanical masker on the processing of information from non-tactile modalities (e.g., audition).

\section{CONCLUSIONS}

Locomotion generates a great deal of multisensory information about walked-upon objects. To date, little or no information is available concerning either our ability to use this information to get to know the environment or the role of different non-visual sensory modalities in the shaping of such a knowledge. This study measured the identification of walking-ground materials in auditory, kinesthetic, haptic and audio-haptic conditions. A novel method was developed to selectively perturb tactile ground-identity information while leaving the kinesthetic information intact.

In line with previous studies of manually-explored objects, haptic perception was in general more accurate than auditory perception. Haptic superiority was potentially the product of the multisensory nature of this modality because the perturbation of tactile information in the kinesthetic condition impaired perceptual performance down to the level observed for the unimodal auditory condition. In line with the hypothesis of a greater multisensory focus on the most accurate information (Welch and Warren, 1980), the analysis of sensory dominance for the identification of solid grounds gave partial evidence in support of a dominance of the most accurate haptic information. In contrast with the same hypothesis, the audio-haptic identification of aggregate materials appeared to focus on the least accurate kinesthetic information. The dominance of kinesthetic information appeared to be a product of biases, and was not explained by discrimination processes. A bias towards focusing on kinesthetic information could be consistent with an optimal decision-making strategy (Kording and Wolpert, 2006) in which individuals attempt to minimize a measure of the expected loss as a result of a potential fall. 


\section{Acknowledgments}

The authors wish to thank F. Gregory Ashby for feedback on GRT fitting procedures, and two anonymous reviewers for useful comments on previous versions of this paper. This work was supported by the Canada Research Chair in Music Perception and Cognition, a grant from the Natural Sciences and Engineering Research Council of Canada (NSERC, RGPIN 312774-05), and a Special Research Opportunity Grant from NSERC to S. McAdams, by a grant to J.R. Cooperstock from the Québec Ministère du Développement Economique, de l'Innovation et de l'Exportation (MDEIE) associated with the European Community $7^{\text {th }}$ Framework Program project Natural Interactive Walking (NIW, no. 222107), and by a grant to V. Hayward from NSERC (RGPIN 6670-07).

Altinsoy, M. (2008). "The Effect of Auditory Cues on the Audiotactile Roughness Perception: Modulation Frequency and Sound Pressure Level," in Haptic and Audio Interaction Design (HAID 2008 Proceedings), edited by A. Pirhonen and S. Brewster (Springer-Verlag, Berlin Heidelberg, Germany), vol. 5270 of Lecture Notes in Computer Science, pp. $120-129$.

ANSI (1974). "Method for the measurement of real-ear protection of hearing protectors and physical attenuation of earmuffs (ANSI S3.19)," Tech. rep., American National Standards Institute, New York, NY.

Ashby, F. G. (1992). Multidimensional Models of Perception and Cognition (Lawrence Erlbaum Associates, Hillsdale, NJ).

Ashby, F. G. and Perrin, N. A. (1988). "Toward a unified theory of similarity and recognition," Psychol. Rev. 95, 124-150.

Ashby, F. G. and Townsend, J. T. (1986). "Varieties of perceptual independence," Psychol. Rev. 93, 154-179.

Avanzini, F. and Crosato, P. (2006). "Haptic-Auditory Rendering and Perception of Contact Stiffness," in Haptic and Audio Interaction Design (HAID 2006 Proceedings), edited by D. McGookin and S. Brewster (Springer-Verlag, Berlin Heidelberg, Germany), vol. 4129 of Lecture Notes in Computer Science, pp. 24-35.

Ballas, J. A. (1993). "Common Factors in the Identification of an Assortment of Brief Everyday Sounds," J. Exp. Psychol. Hum. Percept. Perform. 19, 250-267.

Ballesteros, S. and Heller, M. A. (2008). "Haptic Object Identification," in Human Haptic Perception: Basics and Applications, edited by M. Grunwald (Birkhäuser, Basel, Boston, Berlin), pp. 207-222.

Courtney, A. and Chow, H. M. (2000). "A study of tile design for tactile guide pathways," Int. J. Ind. Ergonomics 25, 693-698.

DiFranco, D. E., Beauregard, G. L., and Srinivasan, M. A. (1997). "The effect of auditory cues on the haptic perception of stiffness in virtual environments," in Proceedings of the ASME dynamic systems and control division, $D S C$, edited by G. Rizzoni (American Society of Mechanical Engineers, U.S., Fairfield, NJ), pp. 17-22.

Donelan, J. M., Kram, R., and Kuo, A. D. (2002). "Mechanical work for step-to-step transitions is a major determinant of the metabolic cost of human walking," J. Exp. Biol. 205, 3717-3727.

Ekimov, A. and Sabatier, J. (2006). "Vibration and sound signatures of human footsteps in buildings," J. Acoust.
Soc. Am. 120, 762-768.

Ekimov, A. and Sabatier, J. (2008). "A Review of Human Signatures in Urban Environments Using Acoustic and Seismic Methods," in Proceedings of IEEE Conference on Technologies for Homeland Security, pp. 215-220.

Ennis, D. M. and Ashby, F. G. (2003). "Fitting decision bound models to identification or categorization data," Tech. rep., Department of Psychology, University of California Santa Barbara.

Ferris, D. P., Louie, M., and Farley, C. T. (1998). "Running in the real world: adjusting leg stiffness for different surfaces," P. Roy. Soc. B-Biol. Sci. 265, 989-994.

Fisher, R. (1915). "Frequency distribution of the values of the correlation coefficient in samples of an indefinitely large population," Biometrika 10, 507-521.

Freed, D. J. (1990). "Auditory correlates of perceived mallet hardness for a set of recorded percussive events," J. Acoust. Soc. Am. 87, 311-322.

Galbraith, F. and Barton, M. (1970). "Ground Loading from Footsteps," J. Acoust. Soc. Am. 48, 1288-1292.

Giordano, B. L., Avanzini, F., Wanderley, M., and McAdams, S. (2010a). "Multisensory integration in percussion performance," in Actes du 10eme Congrès Français d'Acoustique, Lyon (Société Française d'Acoustique, Paris, France), pp. [CD-ROM].

Giordano, B. L. and McAdams, S. (2006). "Material identification of real impact sounds: effects of size variation in steel, glass, wood and plexiglass plates," J. Acoust. Soc. Am. 119, 1171-1181.

Giordano, B. L., McAdams, S., Visell, Y., Cooperstock, J., Yao, H., and Hayward, V. (2008). "Non-visual identification of walking grounds," J. Acoust. Soc. Am. 123, 3412-3412.

Giordano, B. L., Rocchesso, D., and McAdams, S. (2010b). "Integration of acoustical information in the perception of impacted sound sources: The role of information accuracy and exploitability," J. Exp. Psychol. Hum. Percept. Perform. 36, 462-476.

Grassi, M. (2005). "Do we hear size or sound? Balls dropped on plates," Percept. Psychophys. 67, 274-284.

Green, D. M. and Swets, J. A. (1966). Signal detection theory and psychophysics (Wiley, New York, NY).

Guest, S., Catmur, C., Lloyd, D., and Spence, C. (2002). "Audiotactile interactions in roughness perception," Exp. Brain Res. 146, 161-171.

Heller, M. A. (1982). "Visual and tactual texture perception: Intersensory cooperation," Percept. Psychophys. 31, 339344.

ISO (2004). "Acoustics - Reference zero for the calibration of audiometric equipment - Part 8: Reference equivalent threshold sound pressure levels for pure tones and circumaural earphones (ISO 389-8)," Tech. rep., International Organization for Standardization, Geneva.

Jansson, G. (1993). "Perception of the amount of fluid in a vessel shaken by hand," in Studies in Perception and Action II, edited by S. S. Valenti and J. B. Pittinger (Psychology Press, Taylor \& Francis Group, New York, NY), pp. 263-267.

Jones, L. A. and Lederman, S. J. (2006). Human hand function (Oxford University Press).

Jousmäki, V. and Hari, R. (1998). "Parchment-skin illusion: sound-biased touch," Curr. Biol. 8, 190-190.

Kekoni, J., Hämäläinen, H., Rautio, J., and Tukeva, T. (1989). "Mechanical sensibility of the sole of the foot determined with vibratory stimuli of varying frequency," Exp. Brain Res. 78, 419-424. 
Kennedy, P. M. and Inglis, J. T. (2002). "Distribution and behaviour of glabrous cutaneous receptors in the human foot sole," J Physiol (Lond) 583, 995-1002.

Kim, S., Kyung, K., and Kwon, D. (2007). "The Effect of Sound on Haptic Perception," in Second Joint EuroHaptics Conference and Symposium on Haptic Interfaces for Virtual Environment and Teleoperator Systems (WHC'07) (IEEE Computer Society, Los Alamitos, CA), pp. 354-360.

Kinsella-Shaw, J. M., Shaw, B., and Turvey, M. T. (1992). "Perceiving "walk-on-able" slopes," Ecol. Psychol. 4, 223239.

Klatzky, R. L. and Lederman, S. J. (2002). "Touch," in Handbook of Psychology, Volume 4 (Experimental Psychology), edited by A. F. Healy and R. W. Proctor (John Wiley \& Sons, Inc., New York, NY), pp. 147-176.

Klatzky, R. L., Lederman, S. J., and Metzger, V. A. (1985). "Identifying objects by touch: An "expert system"," Percept. Psychophys. 37, 299-302.

Klatzky, R. L., Lederman, S. J., and Reed, C. (1989). "Haptic integration of object properties: Texture, hardness, and planar contour." J. Exp. Psychol. Hum. Percept. Perform. 15, 45-57.

Kobayashi, Y., Osaka, R., Hara, T., and Fujimoto, H. (2008). "How Accurately People can Discriminate the Differences of Floor Materials With Various Elasticities," IEEE Trans. Neural Syst. Rehabil. Eng. 16, 99-105.

Kording, K. and Wolpert, D. (2006). "Bayesian decision theory in sensorimotor control," Trends Cogn. Sci. 10, 319326.

Lederman, S. J. (1979). "Auditory texture perception," Perception 8, 93-103.

Lederman, S. J. and Klatzky, R. L. (2004). "Multisensory texture perception," in Handbook of multisensory processes, edited by G. Calvert, C. Spence, and B. Stein (MIT Press, Cambridge, MA), pp. 107-122.

Lederman, S. J., Klatzky, R. L., Morgan, T., and Hamilton, C. (2002). "Integrating multimodal information about surface texture via a probe: relative contributions of haptic and touch-produced sound sources," in Proceedings of the 10th Symposium on Haptic Interfaces for Virtual Environment and Teleoperator Systems (HAPTICS 2002), pp. $97-104$.

Li, X., Logan, R. J., and Pastore, R. E. (1991). "Perception of acoustic source characteristics: Walking sounds," J. Acoust. Soc. Am. 90, 3036-3049.

Lin, L. I. (1989). "A concordance correlation coefficient to evaluate reproducibility," Biometrics 45, 255-268.

Littell, R. C., Milliken, G. A., Stroup, W. W., Wolfinger, R. D., and Schabenberger, O. (2006). SAS ${ }^{\circledR}$ for mixed models (SAS Institute Inc., Cary, NC), 2 ed.

Lutfi, R. A. (2007). "Human Sound Source Identification," in Auditory Perception of Sound Sources, edited by W. A. Yost, R. R. Fay, and A. N. Popper (Springer, New York, NY), pp. 13-42.

Martin, F. N. and Champlin, C. A. (2000). "Reconsidering the limits of normal hearing," J. Am. Acad. Audiol. 11, 64-66.

Pan, N., Wu, J., Williams, K., and D. Y. Wang, D. (2005). "Effects of Floor Coverings on Posture Steadiness and Locomotion Stability," Tech. rep., National Textile Center.

Pastore, R. E., Flint, J. D., Gaston, J. R., and Solomon, M. J. (2008). "Auditory event perception: The sourceperception loop for posture in human gait," Percept. Psychophys. 70, 13-29.

Perry, S. D., Santos, L. C., and Patla, A. E. (2001). "Con- tribution of vision and cutaneous sensation to the control of centre of mass (COM) during gait termination," Brain Res. 913, 27-34.

Pittenger, J. B., Jordan, J., Belden, A., Goodspeed, P., and Brown, F. (1997). "Auditory and Haptic information support perception of size," in Studies in Perception and Action $I V$, edited by M. A. Schmuckler and J. M. Kennedy (Lawrence Erlbaum Associates, Inc., Mahwah, NJ), pp. 103-105.

Pittenger, J. B. and Mincy, M. D. (1999). "Haptic and auditory information support perception of size: Fine granules," in Studies in Perception and Action V, edited by M. A. Grealy and J. A. Thomson (Lawrence Erlbaum Associates, Inc., Mahwah, NJ), pp. 68-71.

Reyes-Lecuona, A. and Cañadas-Quesada, F. J. (2009). "Interference of Auditory Information with Haptic Perception of Stiffness in Virtual Reality," in Engineering the user interface, edited by M. Redondo, C. Bravo, and M. Ortega (Springer-Verlag, London, UK), pp. 45-56.

Rosburg, T. (2008). "Tactile ground surface indicators in public places," in Human Haptic Perception: Basics and Applications, edited by M. Grunwald (Birkhäuser, Basel, Boston, Berlin), pp. 491-499.

Souman, J. L., Frissen, I., Sreenivasa, M. N., and Ernst, M. O. (2009). "Walking straight into circles," Curr. Biol. 19, 1538-1542.

Spence, C. and Shanker, M. U. (2010). "The influence of auditory cues on the perception of, and responses to, food and drink," J. Sens. Stud. 25, 406-430.

Srinivasan, M. A. and LaMotte, R. H. (1995). "Tactual discrimination of softness," J. Neurophysiol. 73, 88-101.

Suzuki, Y., Gyoba, J., and Sakamoto, S. (2008). "Selective effects of auditory stimuli on tactile roughness perception," Brain Res. 1242, 87-94.

Verbeke, G. and Molenberghs, G. (2000). Linear mixed models for longitudinal data (Springer-Verlag, New York, NY).

Visell, Y. (2011). "Walking on virtual ground: physics, perception, and interface design," Ph.D. thesis, Department of Electrical and Computer Engineering, McGill University.

Visell, Y., Giordano, B., Millet, G., and Cooperstock, J. (2011). "Vibration Influences Haptic Perception of Surface Compliance During Walking," PLoS One 6, e17697.

Warren, W. H. and Verbrugge, R. R. (1984). "Auditory perception of breaking and bouncing events: A case study in ecological acoustics," J. Exp. Psychol. Hum. Percept. Perform. 10, 704-712.

Weinstein, S. (1968). "Intensive and extensive aspects of tactile sensitivity as a function of body part, sex, and laterality," in The skin senses, edited by D. R. Kenshalo (Thomas, Springfield, IL), pp. 195-222.

Welch, R. B. and Warren, D. H. (1980). "Immediate perceptual response to intersensory discrepancy." Psychol. Bull. 88, 638-667.

Well, C., Ward, L. M., Chua, R., and Inglis, J. T. (2003). "Regional Variation and Changes With Ageing in Vibrotactile Sensitivity in the Human Footsole," J. Gerontol. A Biol. Sci. Med. Sci. 58, 680-686.

West, B. T., Welch, K. B., and Galecki, A. T. (2006). Linear mixed models: a practical guide using statistical software (Chapman \& Hall/CRC Press, Boca Raton, FL).

Yao, H.-Y. and Hayward, V. (2010). "Design and analysis of a recoil-type vibrotactile transducer," J. Acoust. Soc. Am. 128, 619-627.

Zampini, M. and Spence, C. (2004). "The role of auditory 
cues in modulating the perceived crispness and staleness of potato chips," J. Sens. Stud. 19, 347-363. 